\title{
Estrategias para facilitar las iniciativas de interacción en hablantes asistidos
}

\author{
EMILI SORO y \\ Nadís-Escuela y Funcación L'Espiga \\ CARMEN BASIL \\ Universitat de Barcelona

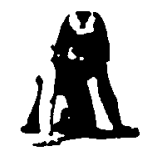 \\ Resumen
}

En este trabajo se estudia la babilidad de iniciar la comunicación en alumnos con parálisis cerebral que usan tableros con pictografías para comunicarse, asi como el papel que juegan los interlocutores en la adquisición de esta babilidad. El estudio expone tres casos, dos niños y una niña de entre 12 y 13 años, escolarizados en un centro de educación especial. Los tres presentan discapacidad motora grave y retraso intelectual. Utilizan tableros de comunicación no electrónicos desde bace 5 años, y sus interlocutores babituales (en casa y en la escuela) ya conocen diferentes estrategias de interacción necesarias para comunicarse con bablantes asistidos. Para facilitar la iniciativa en la comunicación se ba empleado la espera estructurada y la guía física y/o verbal, con la retirada progresiva de estos soportes. Los resultados muestran que tales estrategias, cuando son utilizadas por los interlocutores adultos, pueden aumentar las posibilidades comunicativas de los alumnos. También se ilustra cómo las habilidades aprendidas se mantienen a lo largo del tiempo.

Palabras clave: Parálisis cerebral, Tableros de comunicación pictográficos; iniciativas de interacción; Estrategias de enseñanza; Educaión especial.

\section{Strategies to Facilitate Interaction Initiatives in Aided Speakers}

\section{Abstract}

The ability of children with cerebral palsy to initiate interaction using pictographic communication boards was the focus of this paper togetber with the role played by speech partners in the acquisition of this skill. Three cbildren -2 boys, 1 girl, 12-13 yrs. of age-attending a special school participated in the study. All bad severe motor disabilities and were mentally retarded. The children bad been using non-electronic communication boards for five years when the study began and their usual speech partners (both at bome and at school) were familiar with different types of interaction strategies for communicating with aided speakers. To increase the frequency of children initiating the communication exchange, shaping, imitative and verbal prompts were introduced together with structured waiting tecbniques, and later progressively faded out. Results show that partners can play an important role in promoting children's communication opportunities as the use of such techniques increased the children's ability to actively initiate a conversation. The skills learned were still evidenced in the follow-up study.

Key words: Cerebral palsy; Pictograpbic communication boards; Interaction initiatives; Teaching strategies; Special education.

Agradecimientos: Basado en la ponen cia de Soro, E.; Rey, I.; Reñe, T., y Rossel, C. (1988). Iniciativas de conversación en alumnos que utilizan sistemas aumentativos de comunicación. Presentada en las II Jornadas de G.I.E.E. Octubre. Lleida.

Correspondencia con autores: Nadís, Canàries 1, 08017 Barcelona. 


\section{INTRODUCCION}

Los sistemas aumentativos de comunicación y las ayudas técnicas son un recurso importante para proporcionar a muchas personas con discapacidad de comunicación la posibilidad de interactuar y comunicarse, y también constituyen un recurso para facilitar el acceso a la educación, acceder a actividades de ocio y mejorar la calidad e independencia en la vida diaria. Con estos instrumentos se puede favorecer el proceso de comunicación entendido éste como el intercambio de información que se establece entre dos o más personas, aunque sea con modos y estilos de expresión comunicativa diferentes a los habituales entre personas sin discapacidad. Poyotos (1982, citado en Mathy-Laikko y Yoder, 1986, p. 480) define la comunicación como un «ir $\mathrm{y}$ venir de intercambios que observan ciertas normas, y que también las alteran en el curso irregular de los turnos de interacción entre los parlantes y los oyentes, condicionados por las características personales, por el contexto y la cultura».

A pesar de los avances realizados en el área de la comunicación aumentativa para personas que no pueden usar el habla, se acepra que facilitar al usuario una ayuda técnica (tableros de comunicación, comunicadores electrónicos, ordenadores, etc.) no siempre asegura la comunicación efectiva en el proceso de interacción comunicativa. Como indica Kraat (1985) los sistemas de signos manuales o gráficos son infrautilizados en el proceso cotidiano de comunicación, es decir, que el uso de las ayudas técnicas en ambientes naturales está por debajo de lo que sería posible y deseable. Por otra parte, la descripción de la mayoría de los casos revisados refleja el dominio de la persona oral sobre la no oral en el intercambio comunicativo. Es decir, los adultos ejercen un gran control sobre la interacción y en consecuencia el alumno se ve constreñido en sus opciones comunicativas. El interlocutor parlante produce más intervenciones, mensajes más largos, más diversificación de temas, mayor frecuencia en iniciar la comunicación, etcétera.

Gran número de investigaciones (Basil, 1985, 1992; Beukelman y Yorkston, 1982; Kraat, 1985; Light y Collier, 1986) demuestran que la mayoría de usuarios de ayudas técnicas, electrónicas o no, son sujetos pasivos cuando participan en el proceso de interacción, y la comunicación está dominada por los parlantes mientras que las personas no vocales se limitan a contestar a las preguntas de los primeros. Sin duda que las limitaciones atañen a los dos miembros de la díada comunicativa, y por tanto ambos tendrán que aprender estrategias y habilidades especiales. Según cuales sean las pautas de comportamiento del interlocutor hablante el joven hablante asistido puede no tener oportunidad de aprender a iniciar la interacción o cambiar de tema durante el diálogo, habilidades lingüísticas importantísimas para favorecer el deseo y la práctica de comunicarse. En alumnos con discapacidad grave y usuarios de ayudas técnicas no electrónicas o electrónicas sencillas, los interlocutores vocales han de dominar varias estrategias encaminadas a facilitar que el alumno controle el entorno a través de la comunicación, y, especialmente, han de rehuir la extendida tendencia de enseñar a responder en lugar de facilitar que se aprenda a tomar la iniciativa en la interacción comunicativa.

Light, Collier y Parnes (1985) han estudiado las pautas de interacción de niños de 4 a 6 años, con graves afectaciones motoras y nivel de inteligencia y lenguaje comprensivo normal. Entre éstos y sus madres o personas más cercanas se ha constatado que los patrones de comunicación son sincrónicos pero muy asimétricos, es decir, se produce la interacción manteniendo turnos de comunicación, pero los interlocutores inician más veces, ocupan más tiempo de conversación, formulan más requerimientos y demandas y solicitan respuestas muy específicas de los niños. 
En el estudio realizado por Basil $(1985,1992)$ con alumnos de 7 a 10 años con graves afectaciones neuromotoras y retraso mental que utilizaban tableros de comunicación, se evidenció una falta de sincronía en la interacción además de un gran dominio del adulto en los turnos de comunicación. Se puso de manifiesto el alto porcentaje de iniciaciones de los adultos ( $90 \%$ ) relacionado con un bajo porcentaje de iniciaciones de los alumnos (10\%). El número de intervenciones del adulto fue muy superior al del niño, es decir, el adulto ocupó la mayor parte de la actividad comunicativa. El estudio permitió observar que a pesar de la mejora postintervención en cuanto a la sincronía interactiva (diálogo), las iniciativas de los sujetos continuaban siendo mínimas después de la intervención con los interlocutores (padres y educador).

No siempre se consigue que, de una manera espontánea, los usuarios de comunicación aumentativa interactúen eficazmente con su entorno, es decir, inicien la comunicación, mantengan el tema relevante de diálogo, ajusten las nuevas intervenciones al diálogo planteado por el interlocutor, etc. Esto hace patente la necesidad de intervenir utilizando diversas estrategias dirigidas a los alumnos, a los materiales, a las situaciones y a los interlocutores, entre las que destacan las siguientes:

1. Potenciar los deseos de comunicarse de los alumnos flexibilizando y creando un ambiente favorecedor. Se pueden establecer rutinas de actividades, provocar situaciones nuevas, encontrar temas que interesen a los alumnos, etcétera.

2. Prestar atención de forma muy especial a los intentos comunicativos de los alumnos, ya que al no vocalizar o presentar graves dificultades en la emisión de sonidos, sus intentos de comunicarse pueden pasar inadvertidas por los interlocutores. Expresiones faciales, gestos, movimientos del cuerpo o la boca pueden ser las señales más habituales para iniciar la comunicación en estas personas.

3. Facilitar las posibilidades de comunicación del niño, garantizando que los tableros sean lo más adecuados posible a cada alumno y que los tengan siempre a su alcance. Los tableros se han de organizar con léxico apropiado al nivel evolutivo y a la experiencia del alumno, y crear léxicos monográficos propios para cada situación en el aula, patio, comedor, calle, hogar, etcétera.

4. Facilitar modelos efectivos de comunicación con el uso de ayudas técnicas. Los alumnos pueden aprender técnicas, estrategias y contenidos de comunicación al observar a otras personas que también usan sistemas parecidos. Se pueden organizar sesiones de vídeo, encuentros de grupos de usuarios, ercétera.

5. Ofrecer suficientes oportunidades de repetición de los contenidos y las habilidades lingüísticas que se están aprendiendo. Es importante encontrar un equilibrio entre la novedad de las situaciones y la práctica repetida que es necesaria para la adquisición de nuevos conocimientos en algunos alumnos.

6. Concienciar a las personas que están con el niño (padres, educadores, etc.) de que los tableros de comunicación son realmente el medio de que el niño dispone para transmitir mensajes. Con ello se intenta evitar que usen los tableros como si fueran un cuento que hay que aprenderse.

7. Aumentar el número de interacciones hacia el alumno con preguntas abiertas en lugar de preguntas cerradas que sólo implican una respuesta de sí/no.

8. Producir a menudo frases que no obliguen a una respuesta pero que puedan provocar comunicación, como "parece que no podremos salir al patio...". Mantener el hilo de la comunicación con enunciados cortos no concluyentes como «bueno...», «¡ah!...», «a mí no...», «no sé..., no sé», etcétera.

9. Aumentar los intervalos de tiempo o pausas entre los turnos de comunicación de manera que los alumnos tengan oportunidad de intervenir. Se conocen casos 
de personas que usan comunicación aumentativa que necesitan hasta diez, veinte o más segundos para organizar sus respuestas o intervenciones.

10. Crear programas específicos dirigidos a mejorar la habilidad en el uso de sistemas alternativos de comunicación de todos los interlocutores que están con el niño. Establecer sesiones de práctica de uso de los tableros entre los interlocutores adultos y entre adultos y alumnos con la supervisión de una persona experta, visualizar vídeos con ejemplos correctos de comunicación, etcétera.

11. Planificar el trabajo de forma que permita el desarrollo progresivo de las competencias necesarias para llegar a utilizar espontáneamente el lenguaje. Por ejemplo, se puede establecer rutinas de actividades que después se detienen para que el alumno exprese su deseo de reinicio o continuidad, organizar simulaciones de comunicación funcional con guiones prepreparados, etc. Frumkin (1986), sugiere que las técnicas de «role-playing» con un guión escrito específicamente para un usuario puede ser una estrategia efectiva de intervención que favorecerá el desarrollo de funciones pragmáticas y estrategias conversacionales. Estas actividades de «roleplaying» deben constituir siempre una fase previa de preparación para participar en una situación real, como puede ser ir a comprar a la tienda, pedir el menú en un restaurante, solicitar ayuda en la calle, etcétera.

La intervención en el área del uso de comunicación aumentativa ha de estar muy bien fundamentada para evitar errores que puedan mermar la eficacia del proceso comunicativo, y garantizar que la interacción se convierta en un medio de comunicación y de aprendizaje del lenguaje. La enseñanza debe combinar aspectos estructurales, técnicos, situacionales, etc., pero muy especialmente debe incluir estrategias que fomenten la interacción entre usuarios de sistemas de comunicación alternativa y entre éstos y los interlocutores orales, en sus entornos naturales. Una estrategia crítica de la comunicación, que necesita una atención específica, es la habilidad de iniciar la comunicación. Como apuntan Basil y Ruiz (1988, p. 79) «es difícil planificar el uso espontáneo del léxico, porque precisamente consideramos que la comunicación ha sido espontánea cuando no la hemos solicitado de forma directa o indirecta. Lo que sí podemos planificar son unos aprendizajes previos y crear unas condiciones de interacción que la favorezcan». Para los usuarios de sistemas alternativos, la habilidad de iniciar con frecuencia la interacción comunicativa se considera un objetivo específico que hay que desarrollar (Kraat, 1986). Con tal fin, para el presente estudio se ha elaborado un programa de trabajo encaminado a que los alumnos utilicen de forma espontánea los sistemas aumentativos de comunicación, es decir, que aumenten el número de iniciativas espontáneas de comunicación.

Para posibilitar este objetivo, se aplicaron estrategias que se han comprobado útiles para la enseñanza inicial de comunicación en general, y específicamente en comunicación aumentativa, como son (a) la espera estructurada (Basil, Soro y Von Tetzchner, 1992; Basil y Ruiz, 1988; Light, 1988; Reichle y Sigafoos, 1991; Von Tetzchner y Martinsen, 1993), y (b) la guía física y/o verbal con retirada progresiva de estos soportes (Sigafoos, Mustonen, DePaepe, Reichle y York, 1991; Scott y Reichle, 1991). Con el uso de estas estrategias se pretende que la educadora de los alumnos modifique sus pautas de interacción habituales y que ello conlleve un aumento de iniciativa en la comunicación por parte de los alumnos, utilizando sus tableros de comunicación u otros modos de expresión.

Por iniciación espontánea de la comunicación entendemos la capacidad de llamar la atención del interlocutor para comenzar a hablar de un tema y/o interrumpir un diálogo para abordar un tema nuevo. En este trabajo se han definido como iniciación espontánea del alumno las siguientes actividades: 
TABLA I

Descripción de los sujetos

\begin{tabular}{|c|c|c|c|c|c|c|c|c|c|c|c|}
\hline Sujeto & EC & $\begin{array}{l}\text { Nivel } \\
\text { curri- } \\
\text { cular }\end{array}$ & $\begin{array}{l}\text { Diagnóstico } \\
\text { clínico }\end{array}$ & $\begin{array}{c}\text { Sistema } \\
\text { Aumentativo } \\
\text { de comunicación }\end{array}$ & $\begin{array}{l}\text { Ayuda } \\
\text { técnica }\end{array}$ & $\begin{array}{l}\text { Número } \\
\text { símbolos }\end{array}$ & $\begin{array}{l}\text { Tiempo } \\
\text { de } \\
\text { uso }\end{array}$ & $\begin{array}{c}\text { Forma } \\
\text { de } \\
\text { indicación }\end{array}$ & $\begin{array}{c}\text { Funciones } \\
\text { de } \\
\text { comunicación }\end{array}$ & $\begin{array}{l}\text { Contexto } \\
\text { de } \\
\text { interacción }\end{array}$ & $\begin{array}{l}\text { Inter- } \\
\text { locu- } \\
\text { tores }\end{array}$ \\
\hline$B$ & $\begin{array}{l}13 \mathrm{a} \\
8 \mathrm{~m} .\end{array}$ & 5 & $\begin{array}{l}\text { Parálisis } \\
\text { cerebral. } \\
\text { Tretraparesia } \\
\text { mixta. }\end{array}$ & $\begin{array}{l}\text { Expresiones faciales } \\
\text { comunes. } \\
\text { Sonidos. } \\
\text { SPC. }\end{array}$ & $\begin{array}{l}\text { Tablero de } \\
\text { comunicación: } \\
\text { carpeta con hojas } \\
\text { transparentes. }\end{array}$ & 108 & 5 a. & $\begin{array}{l}\text { Directa con } \\
\text { la mirada, } \\
\text { y por búsqueda }\end{array}$ & $\begin{array}{l}\text { Respuesta a } \\
\text { preguntas. } \\
\text { Demandas. }\end{array}$ & $\begin{array}{l}\text { Aula } \\
\text { escolar. }\end{array}$ & Maestra \\
\hline \multirow[t]{2}{*}{$J$} & $\begin{array}{l}12 \mathrm{a} . \\
10 \mathrm{~m} .\end{array}$ & 4 & $\begin{array}{l}\text { Parálisis } \\
\text { cerebral } \\
\text { Tetraparésia } \\
\text { mixta. }\end{array}$ & $\begin{array}{l}\text { Expresiones } \\
\text { faciales comunes. } \\
\text { Gestos comunes } \\
\text { (con la cabeza y } \\
\text { la mirada). }\end{array}$ & $\begin{array}{l}\text { Tablero de } \\
\text { comunicación } \\
\text { tríptico. } \\
\text { Carpeta auxiliar. }\end{array}$ & 255 & 5 a. & $\begin{array}{l}\text { Codificación } \\
\text { numérica e } \\
\text { indicación directa } \\
\text { con la mirada. }\end{array}$ & $\begin{array}{l}\text { Demandas. } \\
\text { Respuesta a preguntas. } \\
\text { Explicar. } \\
\text { Expresar } \\
\text { acontecimientos }\end{array}$ & $\begin{array}{l}\text { Aula } \\
\text { escolar. }\end{array}$ & Maestra \\
\hline & & & & $\begin{array}{l}\text { Vocalizaciones } \\
\text { SPC. } \\
\text { BLISS. } \\
\text { OT }\end{array}$ & & & & & y sentimientos. & & \\
\hline V & $\begin{array}{l}12 \mathrm{a} . \\
6 \mathrm{~m}\end{array}$ & 5 & $\begin{array}{l}\text { Parálisis } \\
\text { cerebral. } \\
\text { Tetraparesis } \\
\text { espástica. }\end{array}$ & $\begin{array}{l}\text { Gestos de uso } \\
\text { común. } \\
\text { Expresiones facilaes. } \\
\text { Vocalizaciones. } \\
\text { SPC. }\end{array}$ & $\begin{array}{l}\text { Tablero de } \\
\text { comunicación } \\
\text { trípctico. }\end{array}$ & 140 & $5 \mathrm{a}$. & $\begin{array}{l}\text { Indicación directa } \\
\text { con un dedo. }\end{array}$ & $\begin{array}{l}\text { Demandas. } \\
\text { Respuesta a preguntas. } \\
\text { Explicar }\end{array}$ & $\begin{array}{l}\text { Aula } \\
\text { escolar }\end{array}$ & Maestra \\
\hline
\end{tabular}


1 Llamar haciendo un sonido vocal o gutural, si el sonido se produce después de un intervalo de tiempo de como mínimo 1 minuto sin que nadie intervenga solicitando o elicitando la comunicación.

2. Introducir un nuevo tema dentro del diálogo.

La conducta de comunicación se observó en el contexto natural de un aula. Se registraron las iniciativas espontáneas de un grupo de tres alumnos, en sesiones de 30 minutos, durante 21 días distribuidos a lo largo de un curso escolar.

\section{METODO}

\section{Sujetos}

Los sujetos de este estudio son tres alumnos, dos niños y una niña, con parálisis cerebral (Tabla I). Asisten a una escuela especial donde reciben enseñanza y atención fisioterapéutica. Begoña ${ }^{1}$ tiene una edad de 13 a. 8 m., Jaime tiene 12 a. $10 \mathrm{~m}$. y Vicente tiene $12 \mathrm{a} .6 \mathrm{~m}$.

Ninguno de ellos puede desplazarse, y necesitan una silla ortopédica, con adaptaciones especiales, para mantenerse correctamente sentados (Figs. 1, 2 y 3). Vicente puede agarrar algunos objetos con poca precisión y puede señalar con el dedo índice de la mano derecha, Begoña y Jaime no pueden utilizar las extremidades superiores.

Controlan esfínteres, si bien todavía persisten accidentes esporádicos. Dependen totalmente del adulto para realizar las actividades cotidianas: comer, vestirse, etcétera.

FIGURA 1

Adaptación para la sedestación de la alumna Begoña

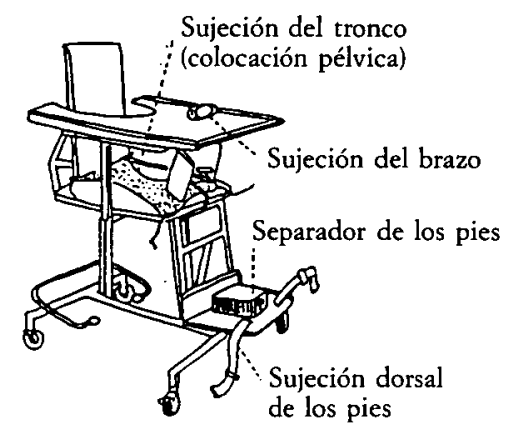

Ninguno de los tres alumnos puede hablar, y usan tableros de comunicación desde hace 5 años. Esta decisión se tomó a partir de una valoración de sus posibilidades de habla y comunicación, basada en los criterios descritos en Soro, Rosell, Alsina, García, Sánchez de Muniaín, Comellas, Vilaseca y Edo (1988), Soro y Marco (1990). Actualmente, los tres alumnos utilizan un tablero de comunicación no electrónico, que permanece siempre sobre su mesa $o$ al alcance de su mirada para que puedan urilizarlo cuando lo necesitan. Los alumnos señalan los signos gráficos en esta ayuda técnica con la mano o la mirada. Los signos gráficos que utilizan se agrupan en los tableros según las posibilidades de percepción e indicación de los alumnos, y teniendo en cuenta criterios de organización sintáctica y semántica del léxico. 
FIGURA 2

Adaptaciones para la sedestación del alumno Jaime

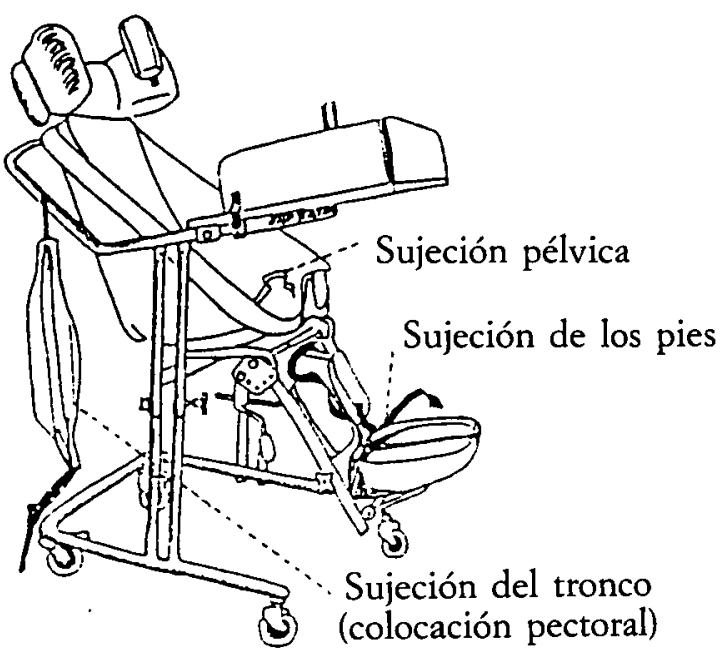

Figura 3

Adaptaciones para la sedestación del alumno Vicente.

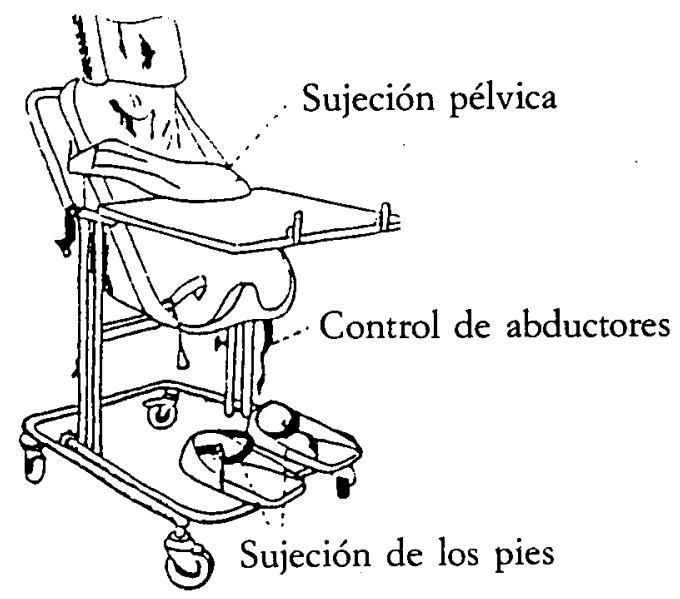

Sujeto 1: Begoña. Begoña utiliza un tablero con fotografías y pictografías SPC tamaño 2,5x2,5 cm (Mayer Johnson, 1981), que consiste en una carpeta con anillas donde se introducen hojas de plástico transparente, tamaño folio, que sirven de soporte a los pictogramas. La carpeta, una vez abierta, deja a la vista 5 signos gráficos con la separación suficiente entre ellos para que los interlocutores vean con claridad qué signo ha mirado la niña. Tiene un vocabulario de 107 signos gráficos que incluyen sustantivos como personas, objetos comunes, lugares, prendas de vestir; verbos de acciones cotidianas como jugar, salir al patio, trabajar, etc.; términos descriptivos como simpático, enfadada, ya está, etc.; términos conversacionales como esto no me gusta; hola, cómo estás; etcétera. 
En la primera página del tablero se sitúan los términos conversacionales, en las siguientes, las personas, las acciones, los nombres diversos y los atributos. La parte superior de las hojas transparentes están señaladas con una cinta adhesiva de color; cada color corresponde a agrupaciones de signos según categorías diversas. La forma principal de indicación de los símbolos se realiza con la mirada (Fig. 4).

FIGURA 4

Tablero de comunicación y forma de indicar de la alumna Begoña.

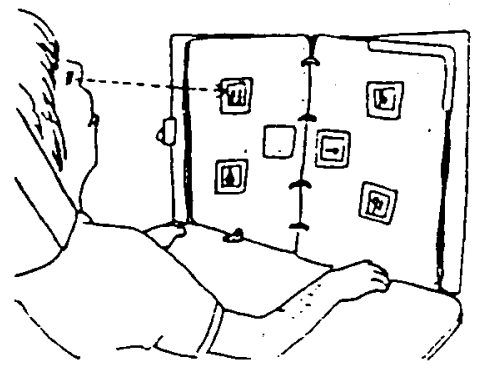

El siguiente es un esquema típico de la mecánica de comunicación con Begoña:

- Alumna: sonido gutural.

- Maestra: Se pone delante de la niña y le dice: «¿Qué quieres?».

- A: Mira el tablero.

- M: Formula preguntas como: «¿Me quieres hablar de la familia?».

- A: Sí (con la cabeza). Cuando responde que «No» se formula una nueva pregunta.

- M: Va directamente a la parte del tablero donde están las personas.

- A: Mira el símbolo «mamá».

- M: «¿Qué ha hecho la mamá?» Va directamente a los verbos y pasa las páginas para que la alumna indique por el sistema de búsqueda en cuál se encuentra lo que quiere explicar.

$-\mathrm{A}$ : «Comer.»

- M: «QQuieres hablarme sobre lo que ha hecho para comer?»

- A: etcétera.

En cuanto a funciones comunicativas, Begoña hace demandas bajo la indicación del adulto y alguna vez de forma espontánea, pero casi siempre pide las mismas cosas, también responde a preguntas sencillas, cerradas y abiertas, y explica acontecimientos presentes, pasados y fururos inmediatos si se le pregunta.

Sujeto 2: Jaime. Jaime utiliza un tablero con fotografías, pictogramas SPC (Mayer Johnson, 1981), Bliss (McDonald, 1985) y ortografía tradicional con palabras escritas en letra mayúscula, dentro de cuadrículas de tamaño $2,5 \times 2,5 \mathrm{~cm}$. El tablero consiste en un tríptico (Basil, Rey, Rosell y Soro, 1988) y carpetas auxiliares. Dispone de un vocabulario de 255 signos gráficos que incluyen sustantivos como personas, objetos comunes, lugares, alimentos, etc.; verbos como mirar, estudiar, esperar, ser, estar, etc.; términos descriptivos como adverbios, adjetivos comunes, términos diversos como preposiciones, partículas, etc.; términos conversacionales como esto sí que es fantástico y tú cómo te encuentras, etc., y las letras del abecedario y los números. 
Indica los símbolos de forma codificada señalando los elementos del código directamente con la mirada. El código consiste en números del $\mathrm{O}$ al 9, estratégicamente situados en el tablero, de manera que cuando el alumno los mira puedan ser identificados por el interlocutor. Cada símbolo tiene asignado un número de un máximo de tres cifras, y se omiten todos los números con cifras repetidas. En cada una de las páginas del tablero tríptico se reproduce la misma secuencia numérica, para evirar una numeración superior a las tres cifras, aunque esto implica que el alumno ha de indicar, antes de iniciar la codificación, a qué página nos tenemos que remitir. Cada secuencia numérica se repite en cada una de las páginas del tablerotríptico (Fig. 5).

Figura 5

Tablero de comunicación y forma de indicar del alumno Jaime.

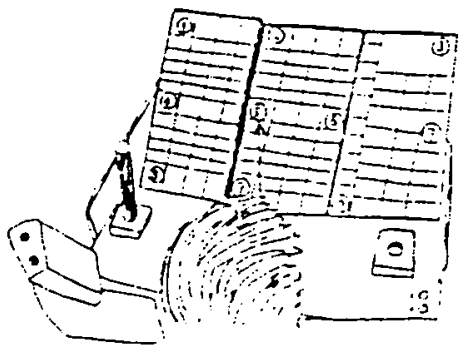

El siguiente es un esquema típico de la mecánica de comunicación con Jaime:

- Alumno: sonido gutural.

- Maestra: ¿Qué quieres? (Siruada delante del alumno.)

- A: Mira una zona del tríptico.

- M: ¿Aquí?

- A: Afirma moviendo los ojos de arriba abajo.

$-\mathrm{M}$ : ¿Primer número?

- A: Mira el número 2.

- M: ¿Segundo número?

- A: Mira el número 5.

- M: Dice la palabra correspondiente al número 25.

En cuanto a las funciones comunicativas, Jaime produce demandas de forma espontánea, responde a preguntas complejas si la respuesta se encuentra dentro de su repertorio expresivo, explica acontecimientos cotidianos presentes, pasados y futuros cuando se lo preguntan. Comienza a explicar acontecimientos y sentimientos personales de forma espontánea. Construye enunciados de dos y tres palabras con algunos interlocutores.

Sujeto 3: Vicente. Vicente utiliza un tablero con pictogramas SPC, tamaño 2,5 $2,5 \mathrm{~cm}$ (Mayer Johnson, 1981), tipo tríptico. Actualmente también utiliza un comunicador electrónico de 16 unidades $\left(\mathrm{MyVoice}^{2}\right)$ con salida de voz digitalizada (voz pregrabada).

Dispone de un vocabulario de 140 símbolos que incluyen sustantivos como personas, objetos comunes, lugares habituales, verbos de acciones cotidianas como salir, comer, bajar, etc.; términos descriptivos como guapo, contento, etcétera.

Indica los símbolos de las ayudas técnicas para la comunicación señalando directamente con el dedo índice de la mano derecha (Fig. 6). 


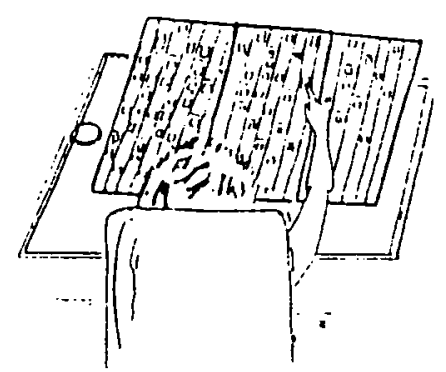

El siguiente es un esquema típico de la mecánica de comunicación con Vicente:

$-A$ : Sonido vocal.

- M: ¿Qué quieres?

- A: Beber, señalando con el dedo.

- M: ¿Qué quieres beber?

- A: Cacao.

En cuanto a las funciones comunicativas, Vicente hace demandas y explica conocimientos presentes y cotidianos bajo la indicación del interlocutor y, esporádicamente, de forma espontánea. Contesta a preguntas sencillas y comienza a emitir enunciados de dos palabras.

\section{Procedimiento}

La maestra conoce las características de comunicación de los niños, y está de acuerdo en mejorar las pautas de interacción. Durante seis sesiones de treinta minutos se pide a la maestra que interactúe con los alumnos en una situación prescrita por el investigador y diseñada con el fin de que ofrezca a estos alumnos buenas oportunidades para iniciar la comunicación. Por ejemplo, se pide a la maestra que exponga materiales y productos que sabe que interesan a los alumnos y anuncie pausadamente, a la vez que señala cada uno de los elementos y los pictogramas correspondientes sobre los tableros, lo siguiente:

- «Como cada día podemos pedir cosas.»

- «Aquí tenemos galletas, refrescos, cacao, podemos ver diapositivas, escuchar un cuento en el casete, jugar con el tren eléctrico, mirar fotos...»

- «¿De acuerdo?"

- «Llamar y pedir lo que queréis."

Sin embargo, no se instruye a la maestra para que haga nada más en especial para fomentar que los niños realmente pidan alguna de estas cosas.

Después de estas sesiones se informa a la maestra de la ventaja que puede comportar el uso de estrategias específicas para «ayudar» a los alumnos a tomar la iniciativa, y se le dan instrucciones sobre las técnicas siguientes:

- La espera estructurada consiste en enseñar una conducta determinada durante un período de tiempo, utilizando las estrategias que se juzguen convenientes; por ejemplo, dando al alumno la oportunidad de responder a las opciones o requerimientos que la maestra ha podido plantear, o guiando su mamo o su mirada en los momentos apropiados, etc. Habituados a esta tarea, la maestra deja de hacer la pregunta o de proporcionar el soporte habitual y espera un tiempo predeterminado para 
dar a los alumnos la oportunidad de emitir una demanda espontáneamente. Por ejemplo, cada día, después de almorzar la maestra dice: «¿Qué queréis ver ahora?", los alumnos pueden señalar en su tablero diapositivas, cuento, álbum de fotos, etc. Más adelante la maestra irá retardando el momento de formular la pregunta, a la espera de la iniciación espontánea del alumno.

- La guía física y/o verbal con retirada progresiva de estos soportes consiste en ofrecer una ayuda al alumno cuando éste no inicia espontáneamente la comunicación. El soporte puede ser de carácter verbal, indicación gestual o guiaje directo de la mirada o la mano del alumno. Combinada con la estrategia anterior, la guía física y/o verbal implica qque durante el intervalo de espera estructurada, cuando el alumno no inicia durante el tiempo preestablecido, se le facilitan soportes graduales hasta conseguir la iniciación. Por ejemplo, después del almuerzo la maestra se mantiene en silencio unos segundos, aparentemente atareada, si los alumnos no inician la comunicación, la maestra puede plantear "podemos ver diapositivas, fotos...", a la vez que indica los signos sobre el tablero de comunicación, o acompaña su mirada o la mano del niño hasta uno de los signos gráficos, si es necesario.

En resumen, durante las diez sesiones de intervención, consecutivas a las seis de línea de base, la secuencia del procedimiento de intervención (Fig. 7) que se ha seguido consiste en:

1. Se organizan una serie de actividades novedosas y de interés para los alumnos. Se establece una rutina en la realización de estas actividades.

2. La maestra espera que el/los alumno/s produzcan alguna indicación (normalmente un sonido). A esta indicación la maestra acude y espera a que el alumno formule una demanda (señalando en su tablero o de otra forma), a lo cual reacciona con comentarios verbales y con la realización de la actividad solicitada.

3. Pasado un minuto sin que los alumnos hayan producido un sonido para iniciar la comunicación, la maestra se les acerca y les hace un gesto interrogativo (una expresión facial o corporal como mover la cabeza, alzar los hombros, etc.) y permanece delante del grupo. Espera hasta que los alumnos producen alguna indicación, durante un intervalo máximo de un minuto.

4. Si después de un minuto los alumnos no han producido ninguna indicación, la maestra les dará un soporte del tipo: «El que quiera alguna cosa que llame», y permanecerá delante del grupo; o...

5. La maestra dirá: «Ahora podéis pedir la/el...», a la vez que lo señala con el dedo; o...

6. La maestra se acerca a uno de los alumnos y guía su mirada o su mano para indicar el signo gráfico sobre su tablero. A esta indicación la maestra responde con comentarios verbales y con la realización de la actividad solicitada.

Después de la intervención se planificaron cinco sesiones de seguimiento pasados dos meses, lo cual completó el diseño del trabajo (Fig. 8), que puede resumirse en las siguientes etapas:

- Observación inicial (Ldb 1), de tres sesiones de 30 min. con la maestra (interlocutora habitual), sin ningún tipo de información referente a las estrategias educativas. Dos observadores registran todas las iniciativas de los tres alumnos.

- Observación inicial repetida (Ldb 2), después de 50 días. Condiciones idénticas a la observación anterior.

- Observación durante la intervención. La maestra ha recibido información de las estrategias educativas específicas. La misma maestra registra las iniciativas de los tres alumnos durante diez sesiones. Dos de las sesiones de intervención también fueron registradas por otro observador. 
- Observación de seguimiento (mantenimiento), después de 120 días de la última sesión de la intervención. Se observaron un total de cinco sesiones en esta etapa de seguimiento.

FIGURA 7

Diagrama del proceso de intervención.

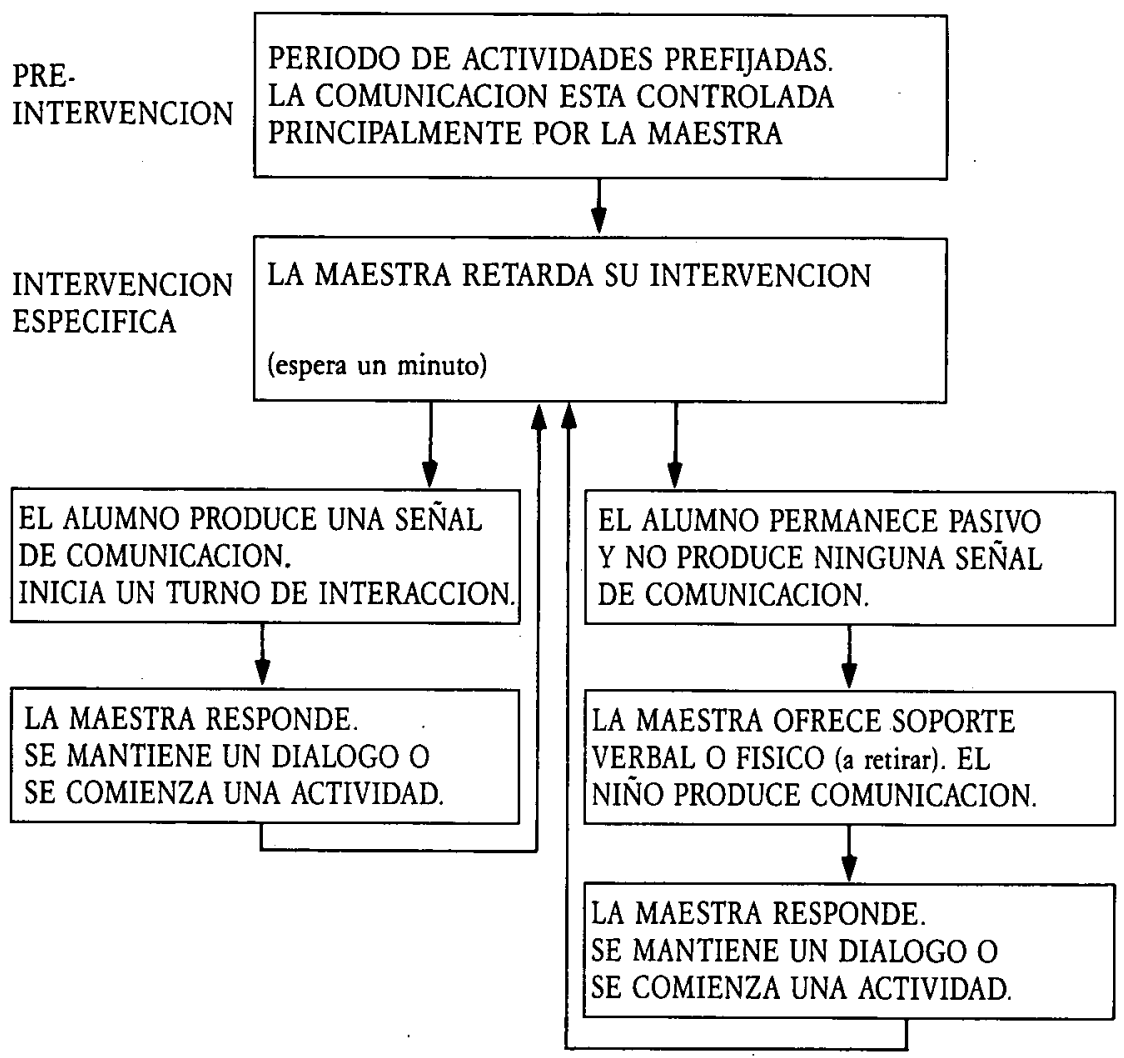

FIGURA 8

Secuencia del procedimientn.

\begin{tabular}{|l|l|l|l|l|l|}
\hline $\begin{array}{l}\text { INTERLO- } \\
\text { CUTORA } \\
\text { no informada }\end{array}$ & $\begin{array}{l}\text { INTERVALO } \\
\text { DE TIEMPO } \\
50 \text { días }\end{array}$ & $\begin{array}{l}\text { INTERLO- } \\
\text { CUTORA } \\
\text { no informada }\end{array}$ & $\begin{array}{l}\text { INTERLO- } \\
\text { CUTORA } \\
\text { informada }\end{array}$ & $\begin{array}{l}\text { INTERVALO } \\
\text { DE TIEMPO } \\
\text { D20 días }\end{array}$ & $\begin{array}{l}\text { INTERLO- } \\
\text { CUTORA } \\
\text { informada }\end{array}$ \\
\hline $\begin{array}{l}3 \text { SESIONES } \\
\text { DE } \\
\text { OBSERVACION }\end{array}$ & & $\begin{array}{l}3 \text { SESIONES } \\
\text { DE } \\
\text { OBSERVACION }\end{array}$ & $\begin{array}{l}\text { 10 SESIONES } \\
\text { DE } \\
\text { OBSERVACION }\end{array}$ &
\end{tabular}


Fiabilidad

La fiabilidad entre observadores: Número de acuerdos dividido por el número total de acuerdos y desacuerdos. Se realizó en cinco sesiones para cada alumno. El acuerdo entre los dos observadores se situó entre un mínimo del $85 \%$ y un máximo del $100 \%$ en las diferentes sesiones/alumnos.

\section{RESULTADOS}

Los datos corresponden a los resultados obtenidos durante un período de un curso escolar, con un total de 21 sesiones de observación, 10 de las cuales fueron de intervención. Durante estas sesiones la maestra tuvo oportunidad de cambiar sus pautas de interacción, las cuales parece que incidieron en que los alumnos iniciaran espontáneamente la comunicación con mayor frecuencia.

Comparando las líneas de base 1 y 2 (Fig. 9) entre las cuales transcurren 50 días, se constata estabilidad entre las observaciones y entre los sujetos. Podemos inferir que el paso del tiempo no tuvo una incidencia importante en la conducta de iniciar la comunicación, y que la conducta era estable.

FIGURA 9

Iniciaciones de los tres alumnos.

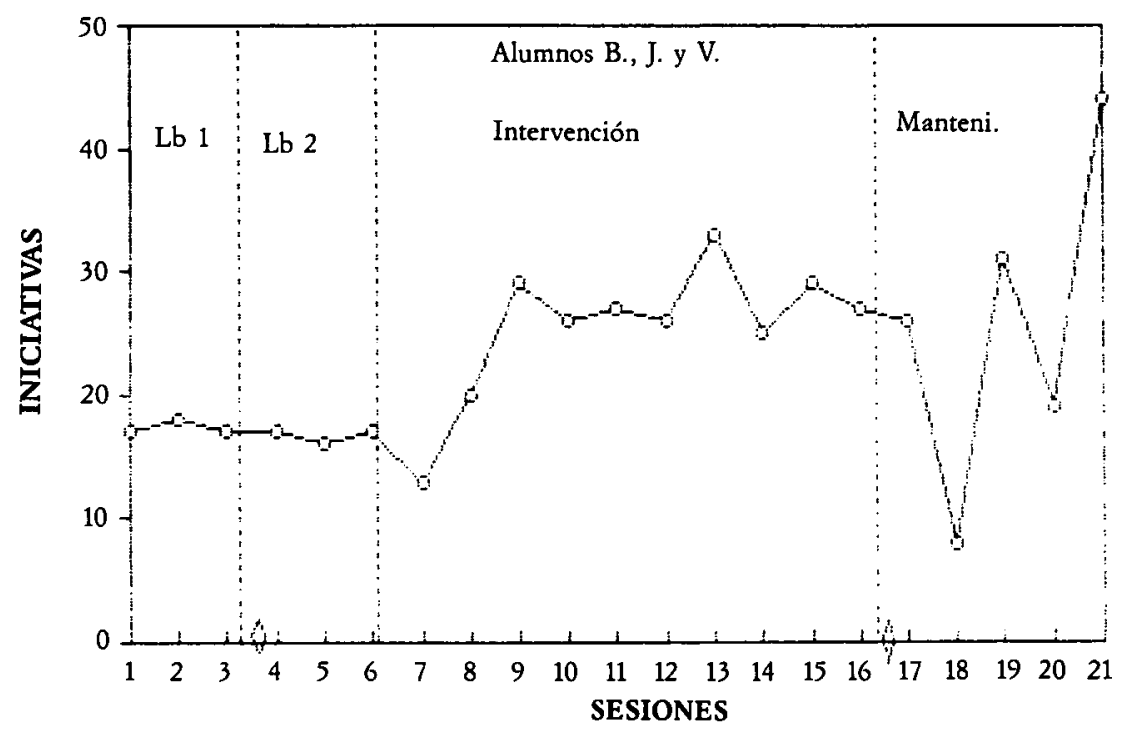

Comparando la frecuencia de iniciativas de las primeras observaciones con el tratamiento, y tal como se había previsto en la hipótesis de trabajo, se observa que el tratamiento parece incidir en el aumento de las demandas espontáneas de los alumnos (Fig. 9). En conjunto, todos los alumnos incrementaron el número de iniciativas a partir de la segunda sesión del tratamiento. En las seis sesiones de línea base (Tabla II) los alumnos inician la comunicación una media de 17 veces, pasando a una media de 25,5 durante las 10 sesiones de intervención educativa, lo que significa un 
aumento del $50 \%$ de iniciativas. In análisis particular de cada sujeto (Figs. 9.1, 9.2. y 9.3) permite observar que la al umna Begoña produce alrededor de 6 iniciativas por sesión, y se mantiene de forma estable a lo largo de los días; Jaime y Vicente se sitúan en torno a las 9 iniciativas en cada sesión, y presentan una marcada inestabilidad según los días. No obstante, cuando se contempla la frecuencia de iniciaciones del grupo de alumnos en las sesiones de la 9 a la 16 (8 últimas semanas de intervención) se observa que se mantiene una alta estabilidad de iniciaciones de los tres alumnos, con una media de 27,75 iniciaciones.

FIGURA 9.1

Iniciaciones de la alumna $B$.

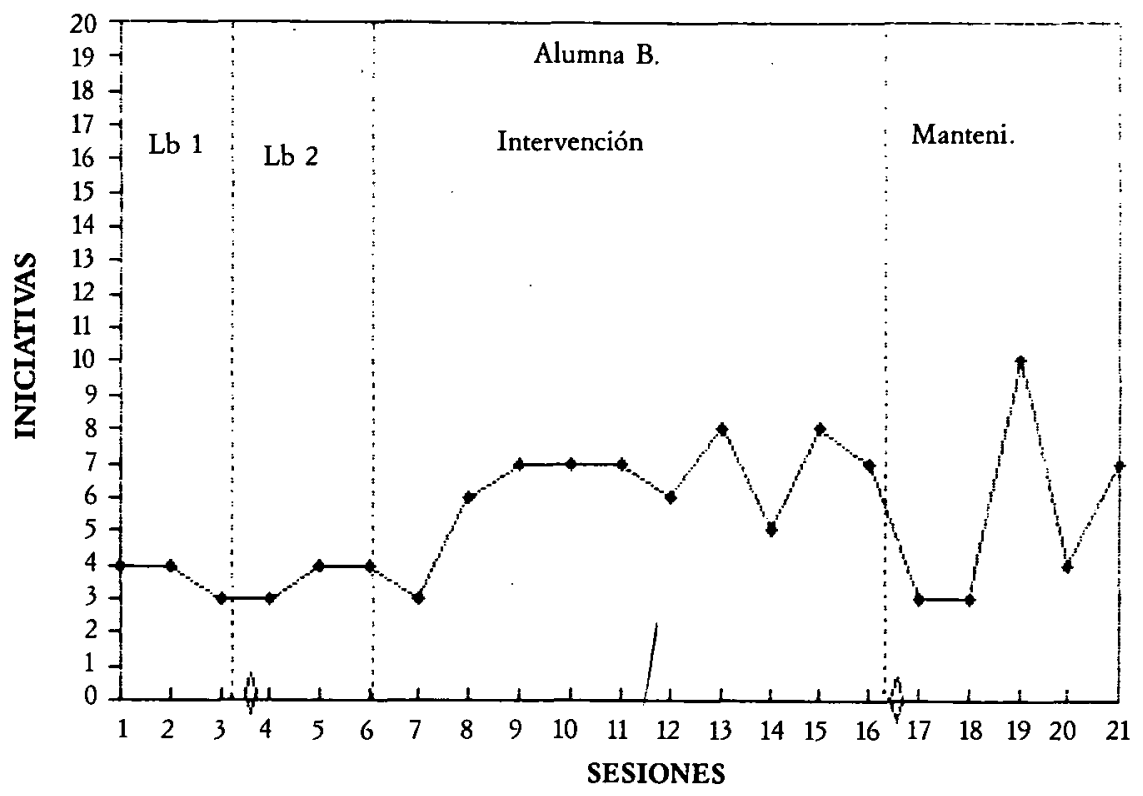

FIGURA 9.2 .

Iniciacimes del alumno. I.

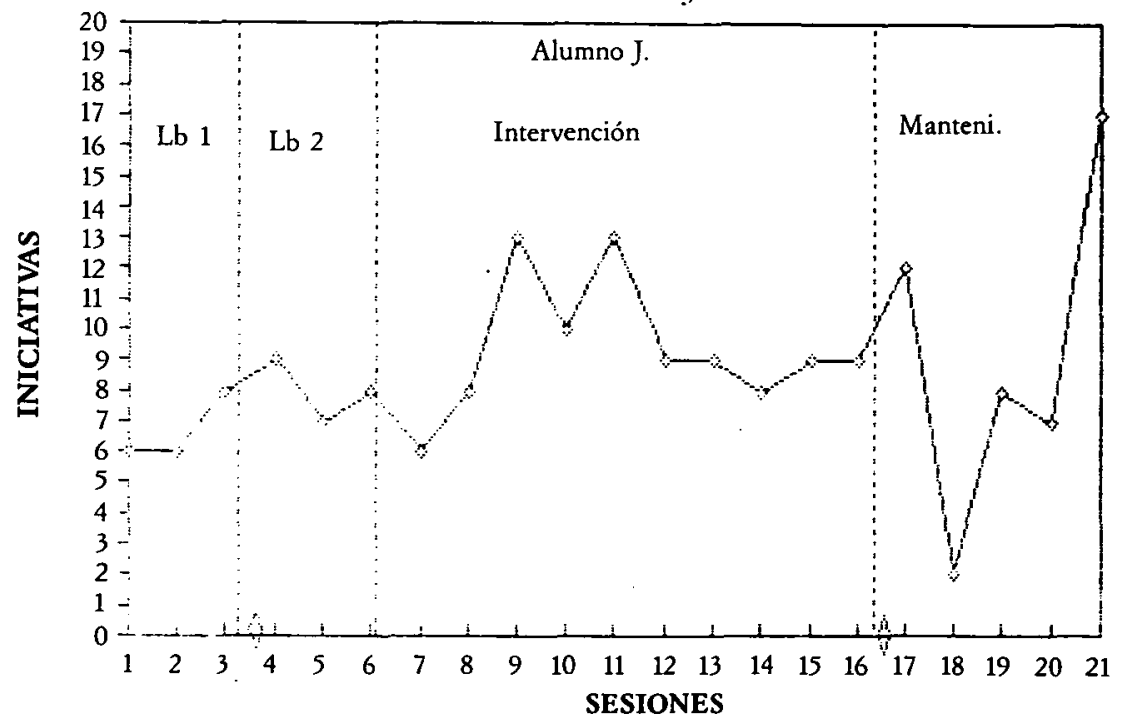


FIGURA 9.3

Iniciación del alumno $\mathrm{V}$

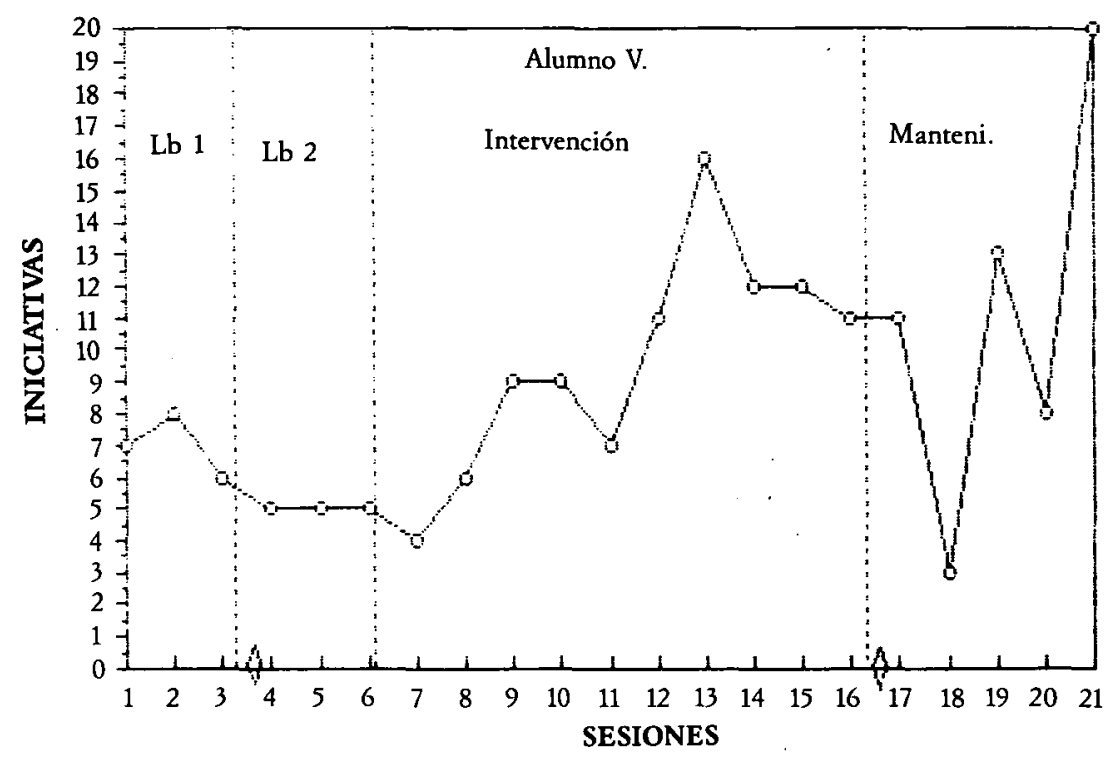

Cuando se compara el tratamiento con el seguimiento, transcurridos 120 días, se constatan grandes variaciones entre sesiones y entre sujetos (Figs. 9, 9.1, 9.2 y 9.3), aunque el promedio de iniciaciones durante esta fase de mantenimiento fue para los tres alumnos más alto que el de la línea de base y prácticamente igual que durante el tratamiento (Tabla II).

TABLA II

Medidas y desviaciones de las iniciativas de los tres alumnos en las diferentes condiciones.

\begin{tabular}{|c|c|c|c|c|}
\hline & B & $\mathrm{J}$ & $\mathrm{V}$ & $\mathrm{B}+\mathrm{J}+\mathrm{V}$ \\
\hline $\mathrm{LB}$ & $\begin{array}{c}X 3,67 \\
\text { SD } 0,52\end{array}$ & $\begin{array}{c}X 7,33 \\
\text { SD } 1,21 \\
\end{array}$ & $\begin{array}{c}\text { X6 } \\
\text { SD } 1,26 \\
\end{array}$ & $\begin{array}{c}X 17 \\
\text { SD } 0,63 \\
\end{array}$ \\
\hline $\mathrm{T}$ & $\begin{array}{c}X 6,40 \\
\text { SD } 1,51\end{array}$ & $\begin{array}{c}X 9,40 \\
\text { SD } 2,17\end{array}$ & $\begin{array}{l}X 9,70 \\
\text { SD } 3,47\end{array}$ & $\begin{array}{l}X 25,50 \\
\text { SD 5,50 }\end{array}$ \\
\hline $\mathrm{M}$ & $\begin{array}{c}X 5,40 \\
\text { SD } 3,05\end{array}$ & $\begin{array}{l}X 9,20 \\
\text { SD } 5,63\end{array}$ & $\begin{array}{c}X 11 \\
\text { SD } 6,28\end{array}$ & $\begin{array}{c}X 25,60 \\
\text { SD } 13,43\end{array}$ \\
\hline
\end{tabular}

\section{DISCUSION}

Este estudio ha ilustrado cómo un reducido conjunto de estrategias educativas pueden ser aplicadas en un contexto natural de aula. Los resultados han sido globalmente positivos, y están en la línea de datos referidos por otros autores, como Schaeffer et al. (1977, citado en Tamarit, 1988) en que un niño autista que muestra una producción de lenguaje espontáneo pretratamiento de 0,17 al día aumenta su producción a 24,37 al día después del tratamiento; o como Light y Collier (1986), 
quienes mencionan el caso de un niño de 4 años no vocal que aumenta el uso de iniciativas espontáneas de 9 , en situación de pretratamiento, a 21 en el postratamiento, en situación de juego libre con su madre, durante diez minutos.

A partir de los resultados cabe resaltar que el objetivo propuesto ha sido alcanzado por los tres sujetos; todos ellos han aumentado el número de iniciativas de comunicación. Podemos decir que las estrategias utilizadas parecen haber sido, en este caso, efectivas. Cuando los interlocutores aplican pautas de interacción adecuadas a los objetivos planteados, los alumnos aprenden a comunicarse mejor. En el objetivo propuesto se evidencia que cuando los adultos hablantes organizan adecuadamente las situaciones educativas, mantienen momentos de silencio y ayudan eficientemente a los alumnos no vocales, éstos tienen más oportunidades de iniciar la comunicación. Los tres alumnos incrementan en un $50 \%$ sus iniciaciones de comunicación, situándose en una media de 27,75 de iniciaciones durante las ocho últimas sesiones de intervención. Asumiendo que, en estas sesiones de 30 minutos, la maestra contestó a todas las intervenciones de los alumnos se infiere que en cada minuto se producían cerca de dos turnos de comunicación, de los cuales la mitad estaban controlados por los alumnos.

En cuanto al análisis individual, los resultados obtenidos y las observaciones clínicas realizadas han puesto de manifiesto una serie de cuestiones que necesitan ser estudiadas en profundidad, como son, por ejemplo, las diferencias individuales de los alumnos, las características y condiciones de los interlocutores, las situaciones educativas y los materiales usados.

En el presente estudio se pone de manifiesto que la estrategia utilizada incrementa el número de iniciativas de los tres alumnos en una situación de grupo. En una situación de grupo como la que se ha estudiado es adecuado que no todos los participantes hablen a la vez, y que por tanto el espacio de comunicación quede repartido entre ellos. Analizando las sesiones de intervención, por ejemplo, las sesiones de la 9 a la 16, se puede decir que todos los participantes intervienen un buen número de veces tomando la iniciativa, pero entre los tres usuarios de comunicación aumentativa se mantienen diferencias acentuadas. Por ejemplo, en las sesiones 9,10 y 11 el alumno Jaime es el que más ha iniciado la comunicación, mientras que en la sesión 12 los tres alumnos han participado con mayor igualdad, y en las últimas sesiones es el alumno Vicente el que ocupa mayor espacio de comunicación. Estos datos inducen a pensar en la necesidad de estudios que analicen las secuencias de interacción dentro del grupo, es decir, que ayuden a dilucidar qué influencia se produce entre los participantes y qué efectos de aprendizaje se producen en la secuencia temporal.

En cuanto a las diferencias individuales se pueden mencionar algunos datos. La diferencia observada entre los diferentes usuarios de comunicación aumentativa que participaron en este estudio se puede explicar en función de las diferencias individuales que existen entre ellos. El alumno Jaime presenta un mayor nivel intelectual y de comunicación, y, al parecer paradójicamente, se observa que no es el que inicia la comunicación con más frecuencia, y que es el más variable en el mantenimiento de esta conducta. Este hecho se puede explicar por el contenido y forma de su lenguaje. Jaime produce enunciados de 2 a 4 palabras, expresa intereses personales variados, ocupa mayor tiempo en mirar un cuento, observando lo que hacen los otros compañeros, etc. Los alumnos Begoña y Vicente, en cambio, llaman a la maestra para pedir un mismo objeto o acción repetidas veces, los enunciados son siempre de una palabra, y el uso del lenguaje se centra en respuesta a preguntas y demandas muy concretas. 
Estos hallazgos están en consonancia con las aportaciones de Light (1988), quien matiza que iniciar no es el único componente de la comunicación competente. Los usuarios de sistemas alternativos de comunicación no inician la comunicación con la frecuencia que lo hacen las personas hablantes, y es cierto que iniciar es importante, pero esto no quiere decir que una alta frecuencia de iniciativas sea, por sí sola, un claro indicador de competencia en la comunicación. El estudio de las funciones comunicativas y del contenido expresado por el usuario cada vez que toma su turno son aspectos tan importante como el anterior, y es un tema que requiere futuros estudios con los usuarios de comunicación aumentativa.

Por otra parte, el adulto interlocutor también puede influir sensiblemente a la creación de un entorno más o menos optimizador de comunicación. Por ejemplo, en la sesión 18 (mantenimiento), la maestra no se encontraba en buenas condiciones de salud, aunque ella insistió en realizar la sesión de observación; los resultados fueron claros, todos los alumnos redujeron drásticamente sus iniciativas.

Merece también atención constatar, aunque ello se aparte del tema específico del análisis que persigue este trabajo, que durante las observaciones no se constató ningún intento de interacción de conversación entre los alumnos. Ni siquiera se observó interacción entre alumnos con la ayuda intermediaria del interlocutor oral. Este es un aspecto poco estudiado y que merece mayor atención educativa en los programas de intervención en alumnos usuarios de comunicación aumentativa.

Los datos recogidos en este estudio se limitan a una cuantificación de la frecuencia de inicio de la comunicación de cada participante, y por tanto no incluyen información sobre el proceso completo de comunicación entre los interlocutores. Esto hace pensar en la necesidad de más estudios en los cuales se analicen los aspectos cualitativos de la comunicación y las características interaccionales de los interlocutores en diferentes contextos. También sería interesante analizar la habilidad de tomar iniciativas de comunicación en relación a otros niveles de competencia lingüística. Aquí se ha estudiado la habilidad de iniciar la comunicación en una situación que permite la expresión de funciones básicas del lenguaje, principalmente, peticiones. Como señalan Keenan y Shieffelin (1976), el objetivo de iniciar la conversación, aunque parezca sencillo, es muy amplio y requiere diferentes pasos que hay que enseñar dentro del proceso global de la comunicación. A saber, el alumno debe aprender a:

- Asegurarse la atención del interlocutor.

- Organizar (articular) el mensaje.

- Facilitar información referente al tema.

- Dar información de la relación entre los referentes del tema.

- Suponer que se espera su discurso, incluso cuando no se le pide claramente respuesta.

- Enseñar a dar una señal cuando han acabado su turno, por ejemplo, señalando un punto (así no se interrumpirá el discurso antes o después de que el alumno lo desee).

- Pedir atención (voz, sonido, aparato).

- Iniciar y cambiar de temas.

- Dar respuestas elaboradas.

- Solicitar que los adultos respondan.

Como es sabido, la comunicación es una conducta especialmente sensible a las variables del entorno y de la propia interacción. Situaciones, materiales, intereses, modos y contenido de la comunicación, estilos personales, etc., pueden incidir en el diálogo comunicativo. Sin obviar que la comunicación es un fenómeno complejo, 
que incluye el uso de complicadas estrategias interrelacionadas, tanto vocales como no vocales y tanto verbales como no verbales, pensamos que el aislamiento de los diferentes factores que lo influyen nos puede ayudar a clarificar la forma de cómo se puede mejorar en su conjunto. El tipo de datos recogidos en este estudio no permite analizar cualitativamente el fenómeno interactivo adulto-alumnos en toda su complejidad, pero sí ha permitido observar un aspecto concreto, aunque no el único relevante, de la comunicación funcional, considerando las habilidades de los usuarios de comunicación aumentativa en un entorno natural y con interlocutores tan significativos como su maestra. Esto debe verse como un primer paso. Todo parece indicar que es necesario potenciar las intervenciones educativas en diversos contextos naturales y en momentos diferentes a lo largo del día, en vez de las intervenciones puntuales en una clase o en sesiones individuales.

Con referencia al propio procedimiento de intervención empleado, como apuntan Von Tetzchner y Martinsen (1993), puede parecer contradictorio establecer rutinas y procedimientos de organización del entorno para fomentar las iniciativas y la espontaneidad de los alumnos. Sin embargo, si se toma la organización del entorno como un medio para facilitar la comunicación y no como un fin en sí mismo, las propias rutinas, aunque pueda parecer contradictorio, suelen incrementar el nivel de actividad y configurar la base para una toma de decisiones más espontánea. Podríamos añadir que la aplicación de estrategias de soporte y ayudas en la educación inicial de una competencia facilita la adquisición de la misma. El problema de la espontaneidad no se soluciona dejando de ayudar al alumno con discapacidad para la comunicación, sino sabiendo retirar debidamente este soporte e ir normalizando, en la medida que lo requieran y lo permitan las necesidades y habilidades del alumno, el proceso de comunicación.

\section{Referencias}

BasI, C. (1985). Procesos d'interacció i comunicació no vocal en infants amb greus afectacions motores. Tesis doctoral inédita. Universidad Autónoma de Barcelona.

BAsIL, C. (1992). Social interaction and learned helplessness in severely disabled children. Augmentative and Alternative Communication, 8(3), 188-199.

BAsIL, C., y RuIz, R. (1988). Sistemes de comunicació no-vocal per a nens amb dismlinucions físiques (2a. Edicion). Barcelona: Documents d'Educació Especial, 3, Generalitat de Catalunya.

BASIL, C.; REY, I.; ROSELL, C., y SORO, E. (1988). Mètode per avaluar i fomentar la comunicació a través d'un plafó. A Generalicat de Catalunya (Ed.). Segones Jornades del Grup d'Investigació en Educació Especial. Barcelona: Documents d'Educació Especial, 9, Generalitat de Catalunya.

BASIL, C.; SORO, E., y VON TETZCHNER, S. (1992). Estrategias iniciales para la ensenyanza de comunicación assistida. (Vídeo). Barcelona: Departament de Psicologia Evolutiva i de l'Educació - ICE Universitat de Barcelona.

Beukelman, D., y Yorkston, K. (1982). Communication interaction of adult communication augmentation system use. Topics in Language Disorders, 2 (2), 39-53.

FRUMKIN, R. J. (1986). Enhancing interaction through role playing. En S. W. Blackstone (Ed.), Augmentative communication: An introduction. Maryland: ASHA.

KEENAN, E. D., y SCHIEFFELIN, B. (1976). Topic as discourse notion: A study of topic in the conversations of children and adults. En: C. N. Li (Ed.), Subject and topic, 335-384. Nueva York: Academic Press.

KraAt, A. W. (1985). Communication Interaction Between Aided and Natural Speakers. A State of the Art Report. Canadá: Canadian Rehabilitation Council for the Disabled.

KRAAT, W. A. (1986). Developing intervention goals. En S. W. Blackstone (Ed.), Augmentative communication: An introduction, 197-266. Maryland: ASHA.

LIGHT, J. (1988). Inceraction Involving Individuals using Augmentative and Alternative Communication Systems: State of the Art and Future Directions. Augmentative and Alternative Communication, 4, 2, 66-82.

LIGHT, J., y COLLIER, B. (1986). Facilitating the development of effective initiation strategies by nonspeaking, physically disabled children. En S. W. Blackstone (Ed.), Augmentative communication: An intraduction, 369-375. Maryland: ASHA. 
Light, J.; Collier, B., y Parnes, P. (1985). Communicative interaction between young nonspeaking physically disabled children and their primary caregivers: Part I - Discourse Patterns. Augmentative and Alternative Communication, 1, 2, 74-83.

MATHY-LAIKKO, P., y YoDER, E. D. (1986). Future needs and directions in augmentative communication. En S. W. Blackstone (Ed.), Augmentative communication: An introduction, 47 1-494. Maryland: ASHA.

MAYER-JOHNson, R. (1981). The picture communication Symbols. Stillwarer: Mayer-Johnson Co. (Se puede obtener en P.O. Box AD, Solana Beach, CA 92075-0838, EE.UU.).

MCDONALd, E. T. (1985). Sistema Bliss: Enseñanza y uso. Madrid: M.E.C. (original inglés en 1980).

Reichle, J., y Sigafoos, J. (1991). Establishing Spontaneity and Generalization. En J. Reichle, J. York y J. Sigafoos (Eds.), Implementing Augmentative and Alternative Communication: Strategies for Leamers with Severe Disabilities, 157-172. Baltimore: Paul H. Brookes. Publishing Co.

ScOTT, L., y ReICHLE, J. (1991). Using Graphic Organization Aids to Promote Independent Functioning. En J, Reichle, J. York y J. Sigafoos (Eds.), Implementing Augmentative and Alternative Communication: Strategies for Learners with Severe Disabilities, 275-288. Balt imore: Paul H. Brookes. Publishing Co.

Sigafoos, J.; Mustonen, T.; DePaepe, P.; Reichle, J., y York, J. (1991). Defining the Array of Instructional Pormpts for Teaching Communication Skills. En J. Reichle, J. York y J. Sigafoos (Eds.), Implementing Augmentative and Alternative Communication: Strategies for Learners with Severe Disabilities, 173-192. Baltimore: Paul H. Brookes. Publishing $C_{0 .}$

Soro, E.; Rosell, C.; Alsina, G.; Garcia, H.; Sanchez de Muniain, P.; Comellas, A.; VilaseCa, R. M., y EDO, S. (1988). Manual de toma de decisiones y de evaluación para el aprendizaje y uso de los sistemas aumentativos de comunicación. Madrid: Serie: evaluación, documento 1. ATAM-FUN DESCO.

Soro, E. y MARCO, F. (1990). Sistemas aumentativos de comunicación como ayuda a la normalización escolar. En CNREE. Las necesidades educativas especiales del niño con deficiencia motora. Seire Formación (Tema 6). Madrid: Publicaciones del CNREE, Ministerio de Educación y Ciencia.

TAmarit, C. J. (1990). Los trastornos de la comunicación en deficiencia mental y otras alceraciones evolutivas: intervención mediante Sistemas de Comunicación Total (2.ed.). En C. Basil y R. Puig (Eds.), Comunicación aumentativa, 137-162. Madrid: Colección Rehabilitación, INSERSO.

VON TETZCHNER, S., y MARTINSEN, H. (1993). Intraducción a la enseñanza de signos y al uso de ayudas técnicas para la comunicación. Madrid: Visor.

\section{Extended Summary}

The present paper studies the ability of children with cerebral palsy to initiate an interaction using pictographic communication boards as well as the role of partners in facilitating children's acquisition of this ability. Three children, two boys and one girl between 12 and 13 years of age with severe motor disabilities and mental retardation, and attending a special school, participated in the study. The study was carried out at their school. The three children had been using communication boards for five years when the study began, and their usual speech partners (both at home and at school) were already familiar with different kinds of strategies to facilitate communication with people using non-electronic pictographic boards. During the study, and in order to increase the frequency of children initiating communication exchanges, shaping, imitative and verbal prompts were introduced together with structured waiting techniques, and later progressively faded out.

Data were collected during 21 sessions spaced across the school year. The first six were baseline sessions, followed by ten intervention sessions, and finally five follow-up sessions. Results showed that speech partners can play an important role in promoting children's communication opportunities since the use of such techniques increases their ability to actively initiate a conservation. During intervention, the teacher had the opportunity to modify her interaction style and this seemed to increase the number of interactions initiated by the children. The follow-up study illustrates how the acquired abilities evolved along a period of time after the intervention had finished. 
The impact of a group situation on communicative interaction between aided speakers is discussed, stressing the need for further studies to achieve a better understanding of interaction sequences between several participants; that is, of the mutual influences taking place along a time sequence and their effects on learning. Individual differences between the three children are also discussed. This leads us to conclude that although the ability to initiate a communication exchange is important for the child, and that aided speakers have difficulty in acquiring this skill, a high level of initiative is not the only index of communicative competence. Communicative functions and content of children's utterances are as important as initiation of communication and they should all be considered as a whole.

Results show a lack of interaction between the three children, even with the help of an adult. This suggests the need for strategies addressed to facilitate interaction between aided speakers. Finally, the specific intervention procedure is discussed, emphasizing the need to develop intervention procedures aimed at facilitating communication in different natural settings. 